\title{
MITTEILUNGEN DER ÖSTERREICHISCHEN GEOGRAPHISCHEN GESELLSCHAFT
}

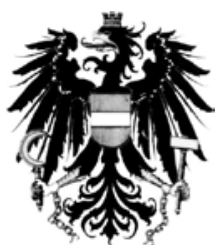

Schriftleitung:

Peter JORDAN 


\begin{abstract}
Die Beiträge in den
„Mitteilungen der Österreichischen Geographischen Gesellschaft“

werden vom Institute for Scientific Information (ISI+Trademarkzeichen;

Philadelphia USA) zur Erstellung des Social Science Citation Index

(SSCI+Trademarkzeichen) regelmäßig referenziert.
\end{abstract}

\title{
Herausgeberkomitee:
}

William Berentsen (Storrs, USA), Axel BorSDORF (Innsbruck), Andre ČERne (Laibach [Ljubljana]), Dušan Drbohlav (Prag [Praha]), Heinz Fassmann (Wien), Derek Hall (Maidens, Scotland, UK), Georg Gartner (Wien), Lorenz Hurni (Zürich), Ioan Ianoş (Bukarest [Bucureşti]), Vladimír Ira (Pressburg [Bratislava]), Károly Kocsis (Budapest), Piotr Korcelli (Warschau [Warszawa]), Sebastian Lentz (Leipzig), Peter Meusburger (Heidelberg), Alexander B. Murphy (Eugene, USA), Ivo Nejašmić (Zagreb), Maria Paola Pagnini (Rom [Roma]), André-Louis Sanguin (Paris), Lothar Schrott (Bonn), Wolfgang Schwarz (Wien), Martin Seger (Klagenfurt a.W.), Christian Staudacher (Wien), Josef Strobl (Salzburg), Hiroshi Tanabe (Tokio [Tōkyō]), Helmut WohlschläGl (Wien), Friedrich ZiMMERMANN (Graz)

Die zu diesem Band eingereichten Manuskripte haben begutachtet:

Hartmut Asche (Potsdam), Mathias BeER (Tübingen), Jozsef BenedeK (Klausenburg [Cluj-Napoca]), Andre ČERne (Laibach [Ljubljana]), Sanda ČORAK (Zagreb), Andreas Dix (Bamberg), Bolesław Domański (Krakau [Kraków]), Heike Egner (Klagenfurt a.W.), Christine Embleton-Hamann (Wien), Heinz Fassmann (Wien), Friedhelm Frank (Dresden), Werner Gamerith (Passau), Daniel Göler (Bamberg), Anton Gosar (Portorož/Portorose), Markus Gruber (Graz), Derek Hall (Maidens, Scotland, UK), Johann HeIss (Wien), Felicitas Hillmann (Erkner, Deutschland), Harald Hitz (Waidhofen a.d. Thaya), Ioan Ianoș (Bukarest [Bucureşti]), Dávid KarÁcsonYI (Budapest), Károly Kocsis (Budapest), Zoltán Kovács (Budapest), Gerhard Karl Lieb (Graz), Přemysl Mácha (Ostrau [Ostrava]), René Matlović (Prešov), Walter Matznetter (Wien), Peter Mayerhofer (Wien), Peter Meusburger (Heidelberg), Jan Mokre (Wien), Heinz Nissel (Wien), Ivan Pirozhnik (Minsk), Denise Pumain (Paris), Hans-Dietrich Schultz (Berlin), Wolfgang Schwarz (Wien), Martin Seger (Klagenfurt a.W.), René Sieber (Zürich), Juraj Silvan (Pressburg [Bratislava]), Armin SKowronek (Bonn), Arthur Spiegler (Wien), Ernst Steinicke (Innsbruck), Ute Wardenga (Leipzig), Doris WastLWalter (Bern), Helmut Wohlschlägl (Wien), Friedrich ZimmermanN (Graz), Jernej Zupanč čc (Laibach [Ljubljana])

Bei personenbezogenen Substantiven gilt in entsprechenden Zusammenhängen die gewählte Form für beide Geschlechter. Dies stellt keine geschlechtsspezifische Diskriminierung dar.

Alle Rechte, auch die der Übersetzung und der fotomechanischen Wiedergabe, sind vorbehalten.

Verleger, Herausgeber und Eigentümer: Österreichische Geographische Gesellschaft, 1070 Wien, Karl-Schweighofer-Gasse 3,

ERSTE Bank Wien 00004405, BLZ 20111; BIC: GIBAATWW, IBAN: AT 502011100000004405

Tel. und Fax: 0043-1-52-37-974 - http://www.oegg.info/

Schriftleitung der „Mitteilungen“:

HR Prof. h.c. Univ.-Doz. Dr. Peter JoRdan, 1010 Wien, Postgasse 7/4/2

Redaktion: HR Prof. h.c. Univ.-Doz. Dr. Peter JoRDAN in Zusammenarbeit mit Karin und Gerhard MAYER (Satz, Layout und Lektorat), und Mag. Walter LANG (Reproduktionstechnik)

Druck: agensketterl Druckerei GmbH, 3001 Mauerbach

ISBN 978-3-901313-28-8

ISSN 0029-9138 